\title{
Solvation in binary mixtures of dipolar hard sphere solvents: Theory and simulations
}

Cite as: J. Chem. Phys. 113, 2360 (2000); https://doi.org/10.1063/1.482051

Submitted: 16 February 2000 . Accepted: 10 May 2000. Published Online: 28 July 2000

M. Morillo, Claus Denk, Francisco Sánchez-Burgos, and Antonio Sánchez

\section{ARTICLES YOU MAY BE INTERESTED IN}

Invariant Expansion for Two-Body Correlations: Thermodynamic Functions, Scattering, and the Ornstein-Zernike Equation

The Journal of Chemical Physics 56, 303 (1972); https://doi.org/10.1063/1.1676864

Invariant Expansion. II. The Ornstein-Zernike Equation for Nonspherical Molecules and an Extended Solution to the Mean Spherical Model

The Journal of Chemical Physics 57, 1862 (1972); https://doi.org/10.1063/1.1678503

Invariant expansion III: The general solution of the mean spherical model for neutral spheres with electostatic interactions

The Journal of Chemical Physics 58, 3295 (1973); https://doi.org/10.1063/1.1679655

\section{Lock-in Amplifiers up to $600 \mathrm{MHz}$}
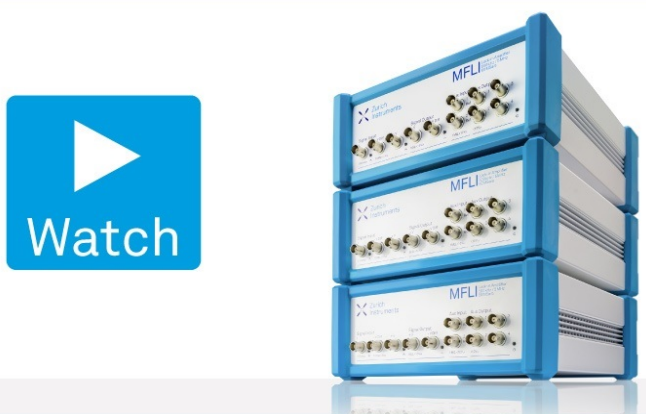

J. Chem. Phys. 113, 2360 (2000); https://doi.org/10.1063/1.482051

113, 2360

(c) 2000 American Institute of Physics. 


\title{
Solvation in binary mixtures of dipolar hard sphere solvents: Theory and simulations
}

\author{
M. Morillo and Claus Denk \\ Universidad de Sevilla, Física Teórica, Apartado 1065, E-41080 Sevilla, Spain \\ Francisco Sánchez-Burgos and Antonio Sánchez \\ Universidad de Sevilla, Química Física, Facultad de Química, C/ Professor García González s/n, \\ E-41012 Sevilla, Spain
}

(Received 16 February 2000; accepted 10 May 2000)

\begin{abstract}
The structure of mixtures of dipolar hard sphere fluids with components of equal size but different dipole moments around a single ion is studied. The solvation energy and the polarization around the ion is obtained in the framework of the mean spherical approximation (MSA). Our theoretical results and the results of other workers are compared with simulation data obtained from Monte Carlo simulations. An interpretation of the meaning of preferential solvation is given in terms of the contrasting behaviors of partial polarization in the bulk and near the ion. (C) 2000 American Institute of Physics. [S0021-9606(00)50830-7]
\end{abstract}

\section{INTRODUCTION}

The solvation of ions by polar solvents has been the subject of numerous theoretical and experimental studies. One of the fundamental quantities is the free energy of solvation. Its simplest description relies on the consideration of the solvent as a macroscopic dielectric continuum that becomes polarized by the presence of the ion. The interaction energy between the solvent polarization and the field created by the ion is then characterized by the dielectric constant of the solvent. Although the macroscopic approximation captures the basic ingredients of the free energy of solvation, their quantitative predictions are often at variance with the experimental findings, in particular for solute ions whose sizes are not very large compared with the size of the solvent molecules.

The limitations of the dielectric continuum treatment of the Born free energy of solvation has led to the introduction of other alternatives that contemplate the molecular description of the solute-solvent system. Starting from the integral equations of liquid theory, and under suitable approximations, analytical expressions for the solvation free energy have been found. In particular, for a model of a hard sphere solute ion in a bath of solvent molecules formed by hard spheres with point dipoles, Chan et al. derived a formula for the Born free energy of solvation within the mean spherical approximation (MSA). ${ }^{1}$ Their expression amounts to replacing the solvent hard sphere diameter in the classical Born expression by an effective one, which depends on the dipole number density, the dipole moment, and the dielectric constant of the pure solvent. Their theoretical treatment gives support to empirical expressions widely used to fit experimental data. A molecular theory of solvation based on the density functional theory has been put forward by Chandra and $\mathrm{Bagchi}^{2}$ leading to an expression for the free energy of solvation which has to be evaluated numerically. Patey and co-workers ${ }^{3}$ have used the linearized hypernetted chain closure (LHNC) to solve the Ornstein-Zernike equation for the same model of hard sphere dipoles embedding a hard sphere point charge. The resulting expressions are more complicated than the corresponding ones found in the MSA, their solutions requiring a nontrivial numerical treatment. It is found that the solvation energy obtained within the LHNC agrees well with that obtained from the MSA, except for solute ions whose diameters are much larger than that of a solvent molecule. Other theoretical approaches are based on the use of perturbation theory. In recent work, Padé approximant techniques have been exploited to truncate the perturbation expansion of the solvation chemical potential of a dipole in dipolar liquids. ${ }^{4}$ Extensions of the dielectric continuum theory that include dielectric saturation and electrostriction effects have been developed in Ref. 5 .

Numerical simulations have also been profusely used in the study of solvation problems. ${ }^{6-12}$ General considerations about the calculation of free energies of solvation can be found in Ref. 13. Although in recent years most of the interest has focused on the analysis of the dynamics of solvation, ${ }^{14-18}$ some work has been devoted to the study of structural and thermodynamical properties. Monte Carlo simulations were used in Ref. 6 to analyze the dependence of the solvation energy in a polar liquid with the solute charge and the influence of dielectric saturation effects. In Ref. 9, the dependence on the ionic charge of hydration free energies of ions is studied. A detailed analysis of the thermodynamics of ion solvation in dipolar fluids using Monte Carlo simulations and the mean reaction field method is presented in Ref. 12. Recently, simulations of a dipole in a bath of polar hard spheres have been presented in Ref. 4 .

In this paper we address the problem of calculating the solvation free energy of an ion in a mixture of polar fluids. Mixtures of polar solvents are very convenient from an experimental point of view as the polarity of the solvent can be easily controlled by varying the composition of the mixture. Nonetheless, the analysis of solvation of ions in mixtures has received less attention than in pure solvents. ${ }^{2,19}$ In a previous 
study, ${ }^{20}$ we analyzed the dependence of the reorganization energy of electron transfer reactions in polar mixtures with the mixture composition, using Monte Carlo techniques. The simulations clearly indicate that there exists an excess reorganization energy: as a small amount of the solvent with higher polarity is added to the mixture, the reorganization energy increases drastically. There, the inability of theoretical expressions to explain this phenomena was noticed. The calculation of the solute-solvent radial distribution function seemed to indicate that the microscopic origin of this phenomenon is the preferential solvation of the ions by the more polar species. The theoretical study of the influence of preferential solvation on the reorganization energy is complex, as one has to deal with two charge centers. Here, we consider the somewhat simpler problem of a single ion in a solution at infinite dilution and carry out a theoretical and simulation analysis of a model system.

We will see that a key ingredient to understand the behavior of the free solvation energy of an ion in a mixture of polar solvents is the preferential solvation of the ion by the component with the higher polarity. The existence of preferential solvation has been related in the literature to deviations of the free solvation energy from an ideal behavior, characterized by a linear dependence of the free energy with the mixture composition. ${ }^{2}$ We believe that deviation from that assumed linearity is not a signature of preferential solvation. Rather, we focus on the behavior of the total and partial polarization densities of the two components around the ion induced by the ion fields. They contain the key solutesolvent correlations and, based on their knowledge, one can give a microscopic interpretation of what is normally termed preferential solvation. Namely, the composition of the mixture near the ion is substantially different from the nominal composition of the liquid in the bulk, away from the ion. This feature can be characterized by the excess local molar fraction parameter (Marcus). ${ }^{21,22}$ The component with the higher polarity contributes to the total polarization density of the first two solvation shells around the ion with a much greater value than its bulk molar fraction would indicate. This local structuration of the mixture in the first few solvation shells gives rise to preferential solvation, and as the authors of Ref. 2 point out, its existence is not necessarily related to specific details of the molecular interactions such as hydrogen bonds. Furthermore, we notice that continuum dielectric theories for the free solvation energy containing the dielectric constant as input are not valid, in general. The reason is that the dielectric constant incorporates just the solvent-solvent correlations in the absence of solute, and these correlations are not the relevant ones in relation with the solvation free energy.

The structure of the paper is as follows. In Sec. II, we present analytical results for a model of a hard sphere solute ion embedded in a mixture of hard sphere dipolar solvents of equal radii and different polarities. The MSA theory for the case of single component solvent has been thoroughly developed by Chan et al. ${ }^{1}$ We extend their ideas to the case of a binary solvent mixture, obtaining analytical expressions for the partial and total solvent polarizations and the free energy of solvation. We also briefly review the theory of Chandra and $\mathrm{Bagchi}^{2}$ based on a linearization of the free-energy functional of an inhomogeneous mixture in the presence of an ion field. In Sec. III we describe the methodology used in our Monte Carlo simulations. Section IV contains the results of the simulations and a comparison with the analytical predictions.

\section{ANALYTICAL THEORIES}

\section{A. MSA for ions in a mixture of hard sphere solvents}

The description of the dielectric properties of solvents within the mean spherical approximation has been carried out by several authors starting from the work of Wertheim. ${ }^{23,24}$ Adelman and Deutch ${ }^{25}$ have solved the problem of a mixture of dipolar hard spheres in the framework of the MSA. In the case of equally sized components, they show that the mixture behaves like a monocomponent fluid with the same hard sphere radius and temperature parameter as the original mixture, but with effective dipole moment

$$
\tilde{\mu}^{2}=\frac{1}{m} \sum_{k} \mu_{k}^{2},
$$

and density

$$
\widetilde{\rho}=\frac{1}{\widetilde{\mu}^{2}} \sum_{k} \mu_{k}^{2} \rho_{k},
$$

where $m$ is the number of components in the mixture, and $\mu_{k}$ and $\rho_{k}$ are the dipole moments and number densities of each component, respectively. The correlation functions are completely determined by the correlation functions of Wertheim's solution for the effective pure polar fluid.

Chan et $a l .{ }^{1}$ have solved the problem of a mixture of ions modeled by hard spheres with point charges in a dipolar solvent of hard spheres with point dipoles, obtaining an expression for the free energy of solvation (Born free energy of charging) of an ion of charge $z e$ at infinite dilution,

$$
F_{B}=-\frac{(z e)^{2}}{2\left(\frac{1}{2} R_{1}+R_{S}\right)}\left(1-\frac{1}{\epsilon}\right) \text {. }
$$

Here, $\epsilon$ is the dielectric constant of the pure dipolar fluid, $R_{1}$ is the ion diameter, and $R_{s}$ is a correction to Born's expression that is given in terms of the dipole radius and the dielectric properties of the pure dipolar fluid.

Our aim is to determine the solvation energy of an ion in a binary mixture of dipolar fluids with equal radii but different dipolar moments. The result of Adelman and Deutch for the solvent mixture in the absence of ions suggests that Eq. (3) could be applied, using the dielectric properties of the effective fluid, as given by Eqs. (1) and (2). In what follows, we will outline the necessary steps to generalize the theory of Chan et al. for a solvent consisting of two components with equal radii and different polarity. We will follow the notation of Chan et al. and will refer to their paper where appropriate.

The ionic solutes are treated as charged hard spheres with diameter $R_{1}$; different species of ions carry charges $z_{\alpha}$ and the electroneutrality condition 


$$
\sum_{\alpha} \rho_{\alpha} z_{\alpha}=0
$$

is assumed to hold, where $\rho_{\alpha}$ denotes the number density for species $\alpha$. The ions are solvated by a mixture of hard spheres, each species having diameter $R_{2}$, number density $\rho_{i}$, and carrying a dipole moment $\mu_{i}$. The interaction potentials have a hard core repulsive term and

$$
\begin{aligned}
& u_{\alpha \beta}(r)=z_{\alpha} z_{\beta} e^{2} / r, \quad r>R_{1}, \\
& u_{\alpha i}\left(\mathbf{r}, \boldsymbol{\omega}_{2}\right)=-z_{\alpha} e \mu_{i} E_{2} / r^{2}, \quad r>R_{12}=\frac{1}{2}\left(R_{1}+R_{2}\right), \\
& u_{i j}\left(\boldsymbol{\omega}_{1}, \mathbf{r}, \boldsymbol{\omega}_{2}\right)=-\mu_{i} \mu_{j} D_{12} / r^{3}, \quad r>R_{2},
\end{aligned}
$$

where we have adopted Greek indices for ions and Latin indices for dipoles. The functions $E_{i}=\hat{\boldsymbol{\mu}}\left(\boldsymbol{\omega}_{\mathbf{i}}\right) \cdot \hat{\mathbf{r}}$ and $D_{i j}$ $=\hat{\boldsymbol{\mu}}\left(\boldsymbol{\omega}_{i}\right)(3 \hat{\mathbf{r}} \hat{\mathbf{r}}-\mathbf{I}) \hat{\boldsymbol{\mu}}\left(\boldsymbol{\omega}_{j}\right)$ are the angular dependent parts of the interaction potentials. In these expressions, $e$ is the elementary charge, $\mathbf{I}$ is the $3 \times 3$ unit tensor, and the unit vector $\hat{\mathbf{r}}$ points from the molecule denoted by the first index towards the one denoted by the second index. The orientation of a dipole moment is defined by the solid angle $\boldsymbol{\omega}$.

The Ornstein-Zernike (OZ) equations for an ion-dipole mixture are given by

$$
\begin{aligned}
& h_{\alpha \beta}(r)=c_{\alpha \beta}(r)+\sum_{\gamma} \rho_{\gamma} \int d \mathbf{s} c_{\alpha \gamma}(|\mathbf{r}-\mathbf{s}|) h_{\gamma \beta}(s) \\
& +\sum_{k} \rho_{k} \int d \mathbf{s}\left\langle c_{\alpha k}(\mathbf{r}-\mathbf{s}) h_{k \beta}\left(\boldsymbol{\omega}_{3}, \mathbf{s}\right)\right\rangle_{\boldsymbol{\omega}_{3}}, \\
& h_{\alpha j}\left(\mathbf{r}, \boldsymbol{\omega}_{2}\right)=c_{\alpha j}\left(\mathbf{r}, \boldsymbol{\omega}_{2}\right)+\sum_{\gamma} \rho_{\gamma} \int d \mathbf{s} c_{\alpha \gamma} \\
& \times(|\mathbf{r}-\mathbf{s}|) h_{\gamma j}\left(\mathbf{s}, \boldsymbol{\omega}_{2}\right)+\sum_{k} \rho_{k} \int d \mathbf{s} \\
& \times\left\langle c_{\alpha k}\left(\mathbf{r}-\mathbf{s}, \boldsymbol{\omega}_{3}\right) h_{k j}\left(\boldsymbol{\omega}_{3}, s, \boldsymbol{\omega}_{2}\right)\right\rangle_{\omega_{3}}, \\
& h_{i \beta}\left(\boldsymbol{\omega}_{1}, \mathbf{r}\right)=c_{i \beta}\left(\boldsymbol{\omega}_{1}, \mathbf{r}\right)+\sum_{\gamma} \rho_{\gamma} \int d \mathbf{s} c_{i \gamma} \\
& \times\left(\boldsymbol{\omega}_{1}, \mathbf{r}-\mathbf{s}\right) h_{\gamma \beta}(s)+\sum_{k} \rho_{k} \int d \mathbf{s} \\
& \times\left\langle c_{i k}\left(\boldsymbol{\omega}_{1}, \mathbf{r}-\mathbf{s}, \boldsymbol{\omega}_{3}\right) h_{k \beta}\left(\boldsymbol{\omega}_{3}, \mathbf{s}\right)\right\rangle_{\omega_{3}}, \\
& h_{i j}\left(\boldsymbol{\omega}_{1}, \mathbf{r}, \boldsymbol{\omega}_{2}\right)=c_{i j}\left(\boldsymbol{\omega}_{1}, \mathbf{r}, \boldsymbol{\omega}_{2}\right) \\
& +\sum_{\gamma} \rho_{\gamma} \int d \mathbf{s} c_{i \gamma}\left(\boldsymbol{\omega}_{1}, \mathbf{r}-\mathbf{s}\right) h_{\gamma j}\left(\mathbf{s}, \boldsymbol{\omega}_{2}\right) \\
& +\sum_{k} \rho_{k} \int d \mathbf{s}\left\langle c_{i k}\left(\boldsymbol{\omega}_{i}, \mathbf{r}-\mathbf{s}, \boldsymbol{\omega}_{3}\right) h_{k j}\right. \\
& \left.\times\left(\boldsymbol{\omega}_{3}, s, \boldsymbol{\omega}_{2}\right)\right\rangle_{\boldsymbol{\omega}_{3}},
\end{aligned}
$$

where $h$ is the total and $c$ the direct correlation function. We have used the notation \langle\rangle$_{\boldsymbol{\omega}}=1 / 4 \pi \int d \boldsymbol{\omega}$ for the angular convolution. In the MSA, the closure conditions are obtained from Eqs. (5)-(7),

$$
\begin{gathered}
h_{I J}\left(\boldsymbol{\omega}_{1}, \mathbf{r}, \boldsymbol{\omega}_{2}\right)=-1, \quad r<R_{I J}^{c}, \\
c_{I J}\left(\boldsymbol{\omega}_{1}, \mathbf{r}, \boldsymbol{\omega}_{2}\right)=-\beta u_{I J}\left(\boldsymbol{\omega}_{1}, \mathbf{r}, \boldsymbol{\omega}_{2}\right) \quad r>R_{I J}^{c} ; \\
I=\alpha, i ; J=\beta, j .
\end{gathered}
$$

Here we have introduced upper case Latin indices which run over both Greek and Latin indices. In this notation the arguments of the correlation functions have to be adjusted depending on the current index: if two Greek indices are present, the argument is $r$. A Greek and a Latin index carry $\mathbf{r}$ and an angular dependence. In this case, if the Latin index is the second one, this angular dependence is $\boldsymbol{\omega}_{2}$, otherwise it is $\boldsymbol{\omega}_{1}$. Two Latin indices carry the full argument as in Eq. (12). The contact distance $R_{I J}^{c}$ is $R_{1}$ for two Greek indices, $R_{2}$ for two Latin indices, and $R_{12}$ for one Greek and one Latin index.

The angular functions $1, E_{i}, D_{i j}$, and $\Delta_{i j}=\hat{\boldsymbol{\mu}}\left(\boldsymbol{\omega}_{i}\right)$ - $\hat{\boldsymbol{\mu}}\left(\boldsymbol{\omega}_{j}\right)$ form a closed set under the angular convolution $\left\langle A\left(\boldsymbol{\omega}_{1}, \boldsymbol{\omega}_{3}\right) B\left(\boldsymbol{\omega}_{3}, \boldsymbol{\omega}_{2}\right)\right\rangle_{\omega 3}$. Multiplication tables for these quantities can be found in Ref. 1. Using this angular decomposition, we use an ansatz of the form

$$
\begin{aligned}
& f_{\alpha \beta}(r)=f_{11}(r)+z_{\alpha} z_{\beta} f^{C}(r), \\
& f_{\alpha j}\left(\mathbf{r}, \boldsymbol{\omega}_{2}\right)=f_{12}(r)+z_{\alpha} \tilde{\mu}_{j} f^{E}(r) E_{2}, \\
& f_{i \beta}(\boldsymbol{\omega} i, \mathbf{r})=f_{21}(r)-z_{\beta} \tilde{\mu}_{i} f^{E}(r) E_{1}, \\
& f_{i j}\left(\boldsymbol{\omega}_{i}, \mathbf{r}, \boldsymbol{\omega}_{2}\right)=f_{22}(r)+\tilde{\mu}_{i} \tilde{\mu}_{j} f^{\Delta}(r) \Delta_{12}+\tilde{\mu}_{i} \tilde{\mu}_{j} f^{D}(r) D_{12}
\end{aligned}
$$

for the correlation functions, where $f=c, h$, and $\tilde{\mu}_{i}$ $=\mu_{i} / \tilde{\mu}$. This conveniently introduces the dipole moment of the effective fluid as defined in Eq. (1). Note that our ansatz is essentially the same as the one in Ref. 1; the angular parts of the correlation functions have been scaled according to the different interaction potentials. The closure conditions (12) in terms of these functions are the hard core conditions $h_{11}$ $=h_{12}=h_{21}=h_{22}=-1$ for distances shorter than contact, and

$$
\begin{aligned}
& c^{C}(r)=-\beta \frac{e^{2}}{r}, \quad r>R_{1}, \\
& c^{E}(r)=\beta \tilde{\mu} \frac{e^{2}}{r^{2}}, \quad r>R_{12}, \\
& c^{D}(r)=\beta \tilde{\mu}^{2} \frac{1}{r^{3}}, \quad r>R_{2} .
\end{aligned}
$$

These conditions are the same as in Ref. 1, when $\mu$ in Ref. 1 is replaced by the dipole moment of the effective pure fluid $\tilde{\mu}$. The three-dimensional Fourier transform

$$
\widetilde{f}\left(\boldsymbol{\omega}_{1}, \mathbf{k}, \boldsymbol{\omega}_{2}\right)=\int d \mathbf{r} e^{\mathbf{i k} \cdot \mathbf{r}} f\left(\boldsymbol{\omega}_{1}, \mathbf{r}, \boldsymbol{\omega}_{2}\right)
$$

of the $\mathrm{OZ}$ equations in our notation may be written as

$$
\begin{aligned}
\widetilde{h}_{I J}\left(\boldsymbol{\omega}_{1}, \mathbf{k}, \boldsymbol{\omega}_{2}\right) \\
=\widetilde{c}_{I J}\left(\boldsymbol{\omega}_{1}, \mathbf{k}, \boldsymbol{\omega}_{2}\right) \\
\quad+\sum_{K} \rho_{K}\left\langle\widetilde{c}_{I K}\left(\boldsymbol{\omega}_{1}, \mathbf{k}, \boldsymbol{\omega}_{3}\right) \widetilde{h}_{K J}\left(\boldsymbol{\omega}_{3}, \mathbf{k}, \boldsymbol{\omega}_{2}\right)\right\rangle_{\boldsymbol{\omega}_{3}} .
\end{aligned}
$$

The angular decomposition of $\widetilde{f}_{I J}\left(\boldsymbol{\omega}_{1}, \mathbf{k}, \boldsymbol{\omega}_{2}\right)$ is the same as in Eq. (13), when $r$ is replaced by $k$ and $f$ by $\tilde{f}$. The 
$k$-dependent angular functions will be denoted by $1, \widetilde{E}_{i}, \widetilde{\Delta}_{i j}$, and $\widetilde{D}_{i j}$. Note that the coefficients of these angular functions may be obtained by one-dimensional Hankel transforms from their $r$-dependent counterparts

$$
\widetilde{f}(k)=4 \pi(-i)^{n} \int_{0}^{\infty} d r j_{n}(k r) f(r),
$$

where $j_{n}(x)$ is the spherical Bessel function of order $n$ and the coefficients are transformed according to

$$
\begin{aligned}
& \widetilde{f}(k)=\widetilde{f}^{D}(k) ; \quad n=2, \\
& \widetilde{f}(k)=\widetilde{f}^{E}(k) ; \quad n=1, \\
& \widetilde{f}(k)=\widetilde{f}^{C}(k), \widetilde{f}^{\Delta}(k), \widetilde{f}_{\alpha \beta}(\alpha, \beta=1,2) ; \quad n=0 .
\end{aligned}
$$

In order to obtain decoupled equations for these quantities, it is convenient to introduce two new orthogonal angular functions

$$
\begin{aligned}
& \widetilde{J}^{+}=1 / 3(\widetilde{\Delta}+\widetilde{D}), \\
& \widetilde{J}^{-}=1 / 3(2 \widetilde{\Delta}-\widetilde{D}),
\end{aligned}
$$

with coefficients

$$
\begin{aligned}
& \widetilde{f}^{+}=\widetilde{f}^{\Delta}+2 \widetilde{f}^{D}, \\
& \widetilde{f}^{-}=\widetilde{f}^{\Delta}-\widetilde{f}^{D} .
\end{aligned}
$$

Carrying out the angular convolution in Eq. (18), using the electroneutrality condition (4), and setting

$$
\begin{aligned}
& \rho_{c}=\sum_{\gamma} \rho_{\gamma} z_{\gamma}^{2}, \\
& \rho_{I}=\sum_{\gamma} \rho_{\gamma}, \\
& \rho_{d}=\sum_{k} \rho_{k},
\end{aligned}
$$

we obtain the following coupled set of equations for the correlation functions:

$$
\begin{aligned}
& \widetilde{h}_{11}(k)=\widetilde{c}_{11}(k)+\rho_{I} \widetilde{c}_{11}(k) \tilde{h}_{11}(k)+\rho_{d} \widetilde{c}_{12}(k) \widetilde{h}_{21}(k), \\
& \widetilde{h}_{12}(k)=\widetilde{c}_{12}(k)+\rho_{I} \widetilde{c}_{11}(k) \widetilde{h}_{12}(k)+\rho_{d} \widetilde{c}_{12}(k) \widetilde{h}_{22}(k) \text {, } \\
& \widetilde{h}_{21}(k)=\widetilde{c}_{21}(k)+\rho_{I} \widetilde{c}_{21}(k) \widetilde{h}_{11}(k)+\rho_{d} \widetilde{c}_{22}(k) \widetilde{h}_{21}(k) \text {, } \\
& \widetilde{h}_{22}(k)=\widetilde{c}_{22}(k)+\rho_{I} \widetilde{c}_{21}(k) \widetilde{h}_{12}(k)+\rho_{d} \widetilde{c}_{22}(k) \widetilde{h}_{22}(k), \\
& \widetilde{h}^{C}(k)=\widetilde{c}^{C}(k)+\rho_{c} \widetilde{c}^{C}(k) \widetilde{h}^{C}(k)-\frac{1}{3} \widetilde{\rho} \widetilde{c}^{E}(k) \widetilde{h}^{E}(k), \\
& \widetilde{h}^{E}(k)=\widetilde{c}^{E}(k)+\rho_{c} \widetilde{c}^{C}(k) \widetilde{h}^{E}(k)+\frac{1}{3} \widetilde{\rho} \widetilde{c}^{E}(k) \widetilde{h}^{+}(k), \\
& \widetilde{h}^{+}(k)=\widetilde{c}^{+}(k)-\rho_{c} \widetilde{c}^{E}(k) \widetilde{h}^{E}(k)+\frac{1}{3} \widetilde{\rho} \widetilde{c}^{+}(k) \widetilde{h}^{+}(k), \\
& \widetilde{h}^{-}(k)=\widetilde{c}^{-}(k)+\frac{1}{3} \widetilde{\rho} \widetilde{c}^{-}(k) \widetilde{h}^{-}(k) \text {. }
\end{aligned}
$$

This set of equations can be divided into three uncoupled groups: group A, Eqs. (30)-(33), together with the corresponding closure conditions (12) defines a mixture of hard spheres of diameter $R_{1}$ at density $\rho_{I}$ and diameter $R_{2}$ at density $\rho_{d}$ with the Percus-Yevick closure. Group B, Eqs.
(35)-(36), describes the angular correlation of the fluid. Finally, Eq. (37) is decoupled from the other equations. When comparing our equations with the results of Chan et al., we note that the angular correlations (group B) are described by the same OZ equations, when $\rho$ is replaced by the density of the effective fluid $\widetilde{\rho}$. As has been noted above, the closure conditions are also identical, replacing $\mu$ by $\tilde{\mu}$. Thus, in what follows, we will use the results of Chan et al. for $\tilde{h}^{+}$ and $\widetilde{h}^{E}$.

The average interaction energy of a single ion with the polar mixture is given by

$$
\begin{aligned}
E & =\frac{1}{4 \pi} \sum_{k} \rho_{k} \iint d \mathbf{r} d \omega u_{\alpha k}(\mathbf{r}, \omega) g_{\alpha k}(\mathbf{r}, \omega) \\
& =-\frac{4 \pi z_{\alpha}^{2} e}{3} \widetilde{\rho} \tilde{\mu} \int_{R_{12}}^{\infty} h^{E}(r),
\end{aligned}
$$

where we have used Eqs. (6) and (13). The Born free energy of solvation for an ion of charge $z e$ is then obtained as ${ }^{1}$

$$
\begin{aligned}
& F_{B}=-\frac{1}{2} \frac{(z e)^{2}}{\frac{1}{2} R_{1}+R_{s}}\left(1-\frac{1}{\epsilon}\right), \\
& R_{S}=R_{2}\left(\frac{1}{2}-\frac{3 \widetilde{\xi}}{(1+4 \widetilde{\xi})}\right),
\end{aligned}
$$

where $\widetilde{\xi}$ is the solution of

$$
Q(2 \widetilde{\xi})-Q(-\widetilde{\xi})=3 \frac{4 \pi}{9} \beta \cdot \widetilde{\rho} \tilde{\mu}^{2} .
$$

Here, $Q(\eta)$ is the Percus-Yevick hard sphere inverse compressibility and $\epsilon$ is the dielectric constant of the pure effective fluid ${ }^{25}$ (see the Appendix). In the following sections, we will compare the predictions of Eq. (39) with the simulation results.

The solvation energy basically depends on the solvent longitudinal polarization density around the ion. The solvent polarization characterizes the response of the solvent to the field of the solvated ion. By definition, the polarization density of the $k$ species in the mixture induced by the ion is given by

$$
P_{k}(r)=\frac{\rho_{k}}{4 \pi} \int d \omega g_{\alpha k}(r, \omega) \boldsymbol{\mu}_{k}(\omega) \cdot \hat{\mathbf{r}}=\frac{\rho_{k} \mu_{k}^{2} z}{3 \tilde{\mu}} h^{E}(r),
$$

and the total polarization density is

$$
P(r)=\sum_{k} P_{k}=\frac{1}{3} \tilde{\rho} \tilde{\mu} z h^{E}(r) .
$$

Far from the ion, the polarization density takes its asymptotic value given by the macroscopic expression

$$
P_{\mathrm{mac}}=\frac{z e}{r^{2}} \frac{1}{4 \pi}\left(1-\frac{1}{\epsilon}\right) \text {. }
$$

It will be illustrative to inspect the ratio $P_{k}(r) / P_{\text {mac }}(r)$. This quantity represents the deviation of the polarization density with respect to the macroscopic model, and it empha- 
sizes the relative contribution of each species. The evaluation of the function $h^{E}(r)$ has been carried out in Ref. 1 and it leads to

$$
\frac{P_{k}(r)}{P_{\mathrm{mac}}(r)}=\frac{\rho_{k} \mu_{k}^{2}}{\widetilde{\rho} \tilde{\mu}^{2}}\left(1-r \frac{d F(r)}{d r}+F(r)\right),
$$

where the quantity $F(r)$ is the solution of an integral equation which can be generated numerically by a trapezoidal rule. The total polarization density can then be written as

$$
\frac{P(r)}{P_{\mathrm{mac}}(r)}=1-r \frac{d F(r)}{d r}+F(r) .
$$

The relative contribution of species $k$ is scaled by the factor $\rho_{k} \mu_{k}^{2}$. For the special case of a binary mixture with $\rho_{1} \mu_{1}^{2} / \rho_{2} \mu_{2}^{2}=1$, both species contribute equally to the total polarization density $P(r)$. These results will be used later on to discuss the meaning of preferential solvation of an ion in a mixture of fluids formed by hard sphere dipoles.

\section{B. The theory of Chandra and Bagchi}

Chandra and $\mathrm{Bagchi}^{2}$ derived an expression for the energy of solvation of an ion in a binary mixture of polar liquids. Their work is based on an expansion of the free-energy functional of the inhomogeneous dipolar mixture in the presence of an external field (the ion field). For the longitudinal polarization of the two species, they obtain

$$
P_{i}(k)=\frac{3}{4 \pi} \gamma_{i}(k) E_{0}(k), \quad i, j=1,2,
$$

where $\mathbf{E}_{0}(\mathbf{k})=E_{0}(k) \hat{\mathbf{k}}$ is the bare electric field of the ion, $E_{0}(k)=i 4 \pi z e j_{0}(k R) / k$, and

$$
\begin{aligned}
& \gamma_{i}(k)=\frac{Y_{i i} X_{j j}(k)-X_{i j}(k) Y_{j j}}{X_{i i}(k) X_{j j}(k)-X_{i j}(k) X_{j i}}, \quad i \neq j, \\
& X_{i j}(k)=\delta_{i j}-\frac{\mu_{i}}{\mu_{j}} \frac{\rho_{i}}{4 \pi} c_{i j}^{m}(110, k), \\
& Y_{i j}=4 \pi \beta \rho_{i} \mu_{i} \mu_{j} / 9 .
\end{aligned}
$$

Here, the $c_{i j}^{m}(110, k)$ are coefficients in the expansion of the wave vector-dependent solvent-solvent direct correlation function in terms of spherical harmonics in the molecular frame (this choice of frame is indicated by the superscript $m) .{ }^{26}$ Namely,

$$
\begin{aligned}
f\left(k, \omega_{1}^{\prime}, \omega_{2}^{\prime}\right)= & \sum_{l_{1} l_{2} m} f^{m}\left(l_{1} l_{2} m, k\right) Y_{l_{1} m}\left(\omega_{1}^{\prime}\right) Y_{l_{2} \bar{m}}\left(\omega_{2}^{\prime}\right), \\
& \tilde{m}=-m .
\end{aligned}
$$

The direct correlation function corresponds to the homogeneous dipolar mixture in the absence of solute ions and it may be obtained in analytical form from the MSA solution of the problem for the effective liquid. ${ }^{25}$ Thus, the coefficients $c_{i j}^{m}(110, k)$ can be readily evaluated within the MSA in terms of the Percus-Yevick solution for a system of nonpolar hard spheres with an effective density which depends on the polarity of the actual solvent.
In what follows, we will restrict ourselves to a mixture of equally sized dipoles with diameter $R=R_{2}$. For this case, the result of Chandra and Bagchi for the solvation energy is

$$
\begin{aligned}
F_{B} & =-\frac{1}{2} \frac{1}{(2 \pi)^{3}} \int d \mathbf{k} \mathbf{P}(\mathbf{k}) \cdot \mathbf{E}_{0}(\mathbf{k}) \\
& =\frac{3(z e)^{2}}{\pi} \int d k\left(\gamma_{1}(k)+\gamma_{2}(k)\right) j_{0}^{2}(k R),
\end{aligned}
$$

and the polarization around the ion is given by

$$
\begin{aligned}
P(r)=P_{1}(r)+P_{2}(r)= & \frac{6 z e}{(2 \pi)^{2}} \int d k k j_{1}(k r) j_{0}(k R) \\
& \times\left(\gamma_{1}(k)+\gamma_{2}(k)\right) .
\end{aligned}
$$

Using the definition of $\gamma_{i}(k)$ and Eq. (A1), it is easy to show that

$$
\frac{P_{1}(k)}{P_{2}(k)}=\frac{\gamma_{1}(k)}{\gamma_{2}(k)}=\frac{\rho_{1} \mu_{1}^{2}}{\rho_{2} \mu_{2}^{2}},
$$

i.e., as in the previous approach, the ratio of the partial polarization densities is fixed by the system parameters. In order to numerically evaluate Eqs. (51) and (52), the coefficients $c_{i j}^{m}(110, k)$ are required. In the Appendix, these functions are given in terms of the Percus-Yevick direct correlation function. The integrands in Eqs. (51) and (52) are difficult to treat numerically. It is convenient to study $\gamma_{12}(k)=\gamma_{1}(k)+\gamma_{2}(k)$ for the limiting cases $k \rightarrow 0$ and $k$ $\rightarrow \infty$ (see the Appendix) and to carry out the integration in three parts, i.e.,

$$
\begin{aligned}
F_{B}= & \frac{3(z e)^{2}}{\pi}\left[\gamma_{12}(0) \int_{0}^{k_{1}} d k j_{0}^{2}(k R)\right. \\
& \left.+\int_{k_{1}}^{k_{2}} d k \gamma_{12}(k) j_{0}^{2}(k R)+\gamma_{12}(\infty) \int_{k_{2}}^{\infty} d k j_{0}^{2}(k R)\right],
\end{aligned}
$$

where the parameters $k_{1}$ and $k_{2}$ are chosen such that the result does not depend on these parameters. The integrals over $j_{0}^{2}\left(k R_{2}\right)$ can be expressed in terms of sine integrals and the numerical integration can now be restricted to a finite range of values $k$ where the integrands are numerically well behaved.

\section{MONTE CARLO SIMULATIONS}

We now turn to the calculation of the solvation free energy from Monte Carlo (MC) simulations of the model system considered in the previous section. Periodic boundary conditions with the minimum image convention ${ }^{27,28}$ were applied to a cubic simulation box of side length $L$. Convergence of the algorithm is accelerated by swapping solvent particles of different species. Details of our simulation method are described in Ref. 20. The simulations presented in this paper have been carried out using $N=863$ solvent particles. We used a packing fraction $\eta=0.42$, corresponding to a dense liquid. A cutoff of 4 molecular diameters has been applied for the long-ranged dipolar forces; contributions from outside the cutoff sphere have been taken into 
account using a generalized reaction field method. ${ }^{29}$ For each system, $10^{8} \mathrm{MC}$ configurations were generated; mean values were calculated after $10^{7}$ equilibration configurations. The dielectric constant of the reaction field has been obtained from simulations of the pure solvent.

The free energy of charging of the solute ion in the solution is given formally by the thermodynamic integration expression $^{30,31}$

$$
\Delta F(0 \rightarrow 1)=\int_{0}^{1} d \lambda\langle V\rangle_{\lambda}
$$

which describes the free-energy difference between the uncharged state of the solute $(\lambda=0)$ and its final charged state with total charge $z e(\lambda=1) . V$ represents the total solutesolvent interaction energy and the angular brackets indicate an average taken with an equilibrium ensemble describing the overall system when the solute charge takes the fictitious value $\lambda z e$. The numerical evaluation of the above expression requires, in principle, the knowledge of the integrand for a large number of points, thus requiring a substantial number of intermediate simulations. As pointed out by Hummer and Szabo, ${ }^{31}$ another alternative to evaluate the free-energy difference is to make use of the information about the statistical distribution of the values of $V$ available from computer simulations of just the initial and final states. Following their analysis, we evaluate the free-energy difference using the expression

$$
\begin{aligned}
\Delta F(0 \rightarrow 1) \approx & \frac{1}{2}\left(\langle V\rangle_{0}+\langle V\rangle_{1}\right)-\frac{\beta}{12}\left(\left\langle\left(V-\langle V\rangle_{0}\right)^{2}\right\rangle_{0}\right. \\
& \left.-\left\langle\left(V-\langle V\rangle_{1}\right)^{2}\right\rangle_{1}\right),
\end{aligned}
$$

which depends on the knowledge of the first moments of the statistical distribution of $V$ in the initial and final states. As shown in Ref. 31, this expression is exact to fourth order in the free-energy perturbation expansion. In order to check the accuracy of Eq. (56), we have carried out simulations for intermediate points; in all cases the corrections to the free energy are found to be within the statistical error limits.

\section{RESULTS AND DISCUSSION}

In Fig. 1 we plot the Born free energy of solvation of an ion with valence $z=1$ as a function of the molar composition for a mixture of two hard sphere dipole liquids with equal radii and dipole moments $\mu_{L}=0.17 \mathrm{e} \AA$ and $\mu_{H}=0.34 \mathrm{e} \AA$. The radius of the solute ion is taken to be the same as the solvent radius, $R_{1} / 2=R_{2} / 2=1.44 \AA$.

Even for the cases of a single component solvent $\left(f_{H}\right.$ $=0$ and $\left.f_{H}=1\right)$, the solvation energy obtained from the MSA and the Chandra and Bagchi theory differs from the value obtained in the simulations. The deviation of the theoretical results from the simulation data is larger for the pure solvent with higher polarity $\left(f_{H}=1\right)$. The simulation results show two main features: first, a steep increase of $F_{B}$ can be noted when a small fraction of high polarity solvent is added to the pure low polarity solvent $\left(f_{H}<0.1\right)$. Second, the value of the free energy of solvation becomes practically independent of the molar fraction for $f_{H}>0.5$. This second feature of

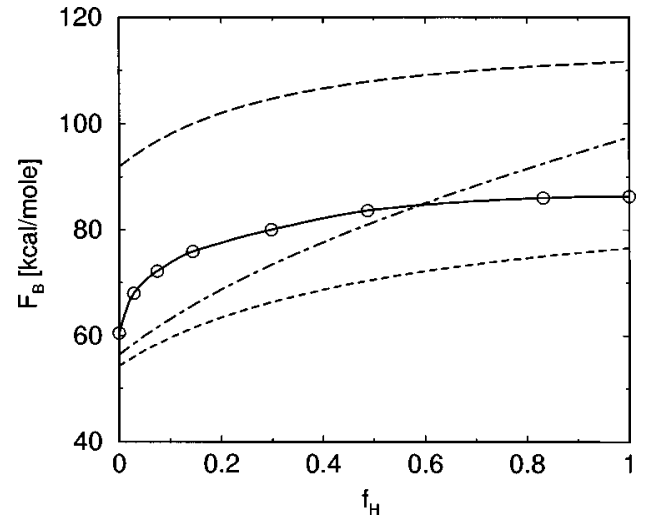

FIG. 1. The Born free energy of solvation for the parameter values given in the main text. The circles are the simulation results; the solid line is an interpolation spline for these points. The dashed line is the theoretical prediction of Eq. (39), the dotted-dashed line is the numerical result obtained from the Chandra and Bagchi approach, Eq. (54). The long dashed line represents the macroscopic Born formula. The variable $f_{H}$ is the molar fraction of the component with higher polarity.

the solvation energy is better described by the MSA results and seems to be absent in the Chandra and Bagchi approach.

Both theories reproduce the deviation of $F_{B}\left(f_{H}\right)$ from an "ideal" law which implies a linear behavior with the molar fraction $f_{H}$. This deviation, as measured by the excess free energy

$$
\Delta F_{B}\left(f_{H}\right)=F_{B}\left(f_{H}\right)-F_{B}(0)-f_{H}\left(F_{B}(1)-F_{B}(0)\right),
$$

has been used in Ref. 2 as an indication for preferential solvation. In Fig. 2 we plot $\Delta F_{B}\left(f_{H}\right)$ for the same parameters as in Fig. 1. In this representation the deviation from the ideal behavior can clearly be seen for both the theoretical and the simulation results. However, the drastic increase of $F_{B}$ for small molar fractions $f_{H}$ (which gives rise to a pronounced asymmetry in $\Delta F_{B}$ ) in the simulation results is not reproduced by the theories. The MSA approach yields a slightly higher value of the excess free energy when compared with the results of Chandra and Bagchi, although both theories underestimate $\Delta F_{B}$.

To further investigate the differences between the simulation results and the theoretical predictions, we now turn our attention to the induced polarization density $P(r)$ around the

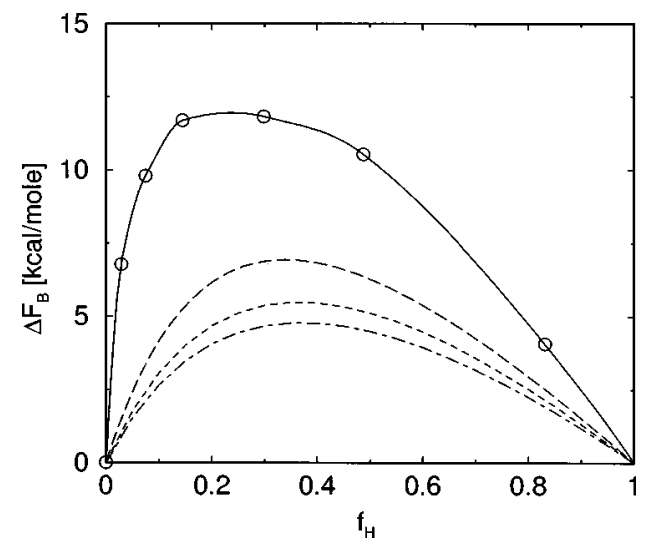

FIG. 2. The excess free energy, Eq. (57), for the same parameters and line types as in Fig. 1. 


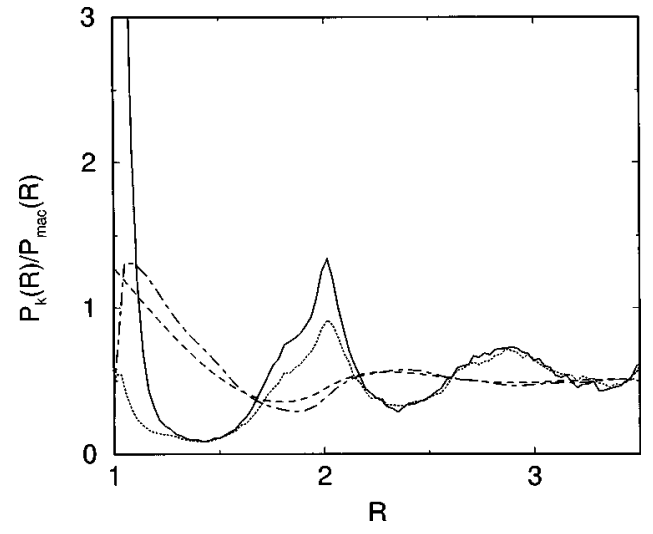

FIG. 3. The partial polarization densities $P_{k}(R) / P_{\mathrm{mac}}(R)$ around the ion for the same system parameters as in Fig. 1. The reduced radius $R$ is defined as $R=r / R_{1}=r / R_{2}$. The composition of the mixture is adjusted by the relation $\rho_{L} \mu_{L}^{2} / \rho_{H} \mu_{H}^{2}=1$, i.e., $f_{H}=0.2$. The simulation results $P_{H}(R)$ and $P_{L}(R)$ are represented by a solid line and a dotted line, respectively. For $\rho_{L} \mu_{L}^{2} / \rho_{H} \mu_{H}^{2}=1$, both theories give $P_{L}(R)=P_{H}(R)$. The MSA result is plotted as a dashed line, while a dotted-dashed line is used for the Chandra and Bagchi approach.

ion. In Fig. 3 we represent the partial polarization densities $P_{L}(r) / P_{\mathrm{mac}}(r)$ and $P_{H}(r) / P_{\mathrm{mac}}(r)$ for the case of a mixture with $\rho_{L} \mu_{L}^{2} / \rho_{H} \mu_{H}^{2}=1$, i.e., $f_{H}=0.2$. For this choice of parameters, the partial polarizations of the low and high polarity species $\left[P_{L}(r)\right.$ and $\left.P_{H}(r)\right]$ obtained within the MSA [see Eq. (44)] are identical. The same holds true for the Chandra and Bagchi approach [see Eq. (53)]. In Fig. 3, this is manifested by the coincidence of the two partial polarization density plots for each theoretical description. Both theoretical results give a similar behavior, except for values of $r$ close to contact. The first maximum of $P_{k}(r)(k=L, H)$ lies at $r$ $=R$ in the MSA method, while the Chandra and Bagchi result shows a first maximum for values $r$ slightly larger than $R$. For values $r \rightarrow \infty$, both theories predict the expected macroscopic bulk value of the polarization density $P_{L}(r) / P_{\text {mac }}(r)=P_{H}(r) / P_{\text {mac }}(r)=1 / 2$.

The failure of the theoretical polarizations to describe the simulation results are clearly seen in the figure. The partial polarization densities $P_{k}^{\mathrm{sim}}(r)$ show oscillations, even for intermolecular distances at which the theoretical results have already reached their asymptotic value. Furthermore, the positions of the maxima and minima are displaced. Thus, the structure of the first few solvation shells is not predicted well by the theories. Another discrepancy manifests itself when studying the first and second solvation shell. While the theories predict $P_{L}(r)=P_{H}(r)$ for all $r$, the simulation indicates that the contribution of $P_{H}$ prevails for small intermolecular distances. This feature is more pronounced for the first solvation shell.

An interesting conclusion that can be drawn from the results discussed above concerns the meaning of preferential solvation. In the work of Chandra and Bagchi, preferential solvation is defined in terms of the deviation of the solvation energy from its ideal behavior with respect to the molar fraction. Let us suppose for a moment that the ion-dipole pair distribution function for species $k=L, H$ is defined as

$$
g_{\alpha k}\left(\mathbf{r}, \omega_{2}\right)=g_{\alpha k}(r)+z \frac{\mu_{k}}{\tilde{\mu}} h_{k}^{E}(r) E_{2}^{k},
$$

i.e., both the radial and the angular component of the correlation function depend on the index $k$. In this case, the angular average of $\boldsymbol{\mu}_{\mathbf{k}} \cdot \hat{\mathbf{r}}$ is given by

$$
\begin{aligned}
\left\langle\mu_{k} E_{2}^{k}\right\rangle(r) & =\frac{\frac{1}{4 \pi} \int d \omega_{2} \mu_{k} E_{2}^{k} g_{\alpha k}\left(\mathbf{r}, \omega_{2}\right)}{\frac{1}{4 \pi} \int d \omega_{2} g_{\alpha k}\left(\mathbf{r}, \omega_{2}\right)} \\
& =\frac{1}{3} z \frac{\mu_{k}^{2}}{\tilde{\mu}} h_{k}^{E}(r) g_{\alpha k}(r),
\end{aligned}
$$

and the partial polarization due to species $k$ is

$$
\begin{aligned}
P_{k}(r) & =\frac{\rho_{k}}{4 \pi} \int d \omega_{2} \mu_{k} E_{2}^{k} g_{\alpha k}\left(\mathbf{r}, \omega_{2}\right) \\
& =\rho_{k} \mu_{k}^{2}\left\langle E_{2}^{k} / \mu_{k}\right\rangle(r) g_{\alpha k}(r)=\rho_{k} \mu_{k}^{2} \hat{h}^{k}\left(r, f_{H}\right),
\end{aligned}
$$

where we have introduced $\hat{h}^{k}\left(r, f_{H}\right)$. The argument $f_{H}$ in this function indicates that $\hat{h}^{k}\left(r, f_{H}\right)$ generally depends on the molar composition of the mixture. The ratio of the partial polarizations will thus be given by

$$
\frac{P_{H}(r)}{P_{L}(r)}=\frac{\rho_{H} \mu_{H}^{2} \hat{h}^{H}\left(r, f_{H}\right)}{\rho_{L} \mu_{L}^{2} \hat{h}^{L}\left(r, f_{H}\right)} .
$$

By writing the partial polarization densities in the form of Eq. (60), we have conveniently separated the $r$-dependent contributions to $P_{k}(r)$ that describe the angular correlations $\left\langle E_{2}^{k} / \mu_{k}\right\rangle(r)$, on one hand, and the radial distribution functions $g_{\alpha k}(r)$ on the other.

In the previous sections, we have shown that for the two theories discussed in this paper, the ratio $P_{H}(r) / P_{L}(r)$ is a constant independent of $r: P_{H}(r) / P_{L}(r)=\rho_{h} \mu_{H}^{2} / \rho_{L} \mu_{L}^{2}$. This arises because in the framework of the two theoretical treatments mentioned in this paper, $\hat{h}\left(r, f_{H}\right)$ has the same functional form for both components of the mixture at a fixed molar fraction, so that $\hat{h}^{H}\left(r, f_{H}\right)=\hat{h}^{L}\left(r, f_{H}\right)=\hat{h}\left(r, f_{H}\right)$. Note that the function $\hat{h}\left(r, f_{H}\right)$ that is implicit in the work of Chandra and Bagchi differs from $\hat{h}\left(r, f_{H}\right)$ in the MSA. The total polarization in terms of $\hat{h}\left(r, f_{H}\right)$ is then

$$
P(r)=P_{L}(r)+P_{H}(r)=\rho_{d}\left(\mu_{L}^{2}+f_{H}\left(\mu_{H}^{2}-\mu_{L}^{2}\right)\right) f\left(r, f_{H}\right) .
$$

If $\hat{h}\left(r, f_{H}\right)$ were independent of $f_{H}$, Eq. (62) would yield a linear (ideal) behavior of the solvation energy with the molar fraction. Thus, the nonideality observed in Fig. 2 is a consequence of the dependence of $\hat{h}\left(r, f_{H}\right)$ on the molar fraction. One should note that, even when using the Born formula for the solvation energy, with a dielectric constant of the mixture evaluated following the prescription of Adelman and Deutch, ${ }^{25}$ a nonideal behavior is obtained. This is to be expected, as the dielectric constant is not a linear function of $f_{H}$. This limited description is not capable of describing the intuitive picture of preferential solvation: when the ion is preferentially solvated by the more polar species, one usually has in mind that the first solvation shell is composed, to a 


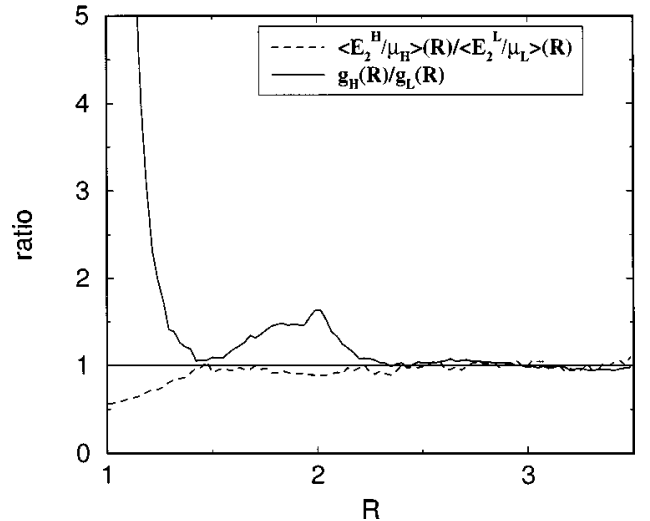

FIG. 4. The ratios $\left\langle E_{2}^{H} / \mu_{H}\right\rangle(R) /\left\langle E_{2}^{L} / \mu_{L}\right\rangle(R)$ and $g_{\alpha H}(R) / g_{\alpha L}(R)$ as obtained from the simulation. The parameters are the same as in Fig. 3.

large extent, of molecules of the more polar species. This has as a consequence that $g_{\alpha H}(r)$ and $g_{\alpha L}(r)$ are different, and the ratio $\hat{h}^{H}\left(r, f_{H}\right) / \hat{h}^{L}\left(r, f_{H}\right)$ is no longer unity and will generally depend on $r$. The simulation results for the partial polarizations shown in Fig. 3 show just this behavior. The ratio $\hat{h}^{H}\left(r, f_{H}\right) / \hat{h}^{L}\left(r, f_{H}\right)$ differs from unity in the first two solvation shells; for distances close to contact we obtain values as high as $\hat{h}^{H}(r, 0.2) / \hat{h}^{L}(r, 0.2) \approx 25$.

It is instructive to analyze the ratios $\left\langle E_{2}^{H} / \mu_{H}\right\rangle$ $\times(r) /\left\langle E_{2}^{L} / \mu_{L}\right\rangle(r)$ and $g_{\alpha H}(r) / g_{\alpha L}(r)$ separately. In Fig. 4 we have plotted these ratios from the simulation data for a molar fraction of $f_{H}=0.2$. It can clearly be seen that the main contribution to the deviation of $\hat{h}^{H}\left(r, f_{H}\right) / \hat{h}^{L}\left(r, f_{H}\right)$ from unity stems from the radial contribution, i.e., from a spatial reorganization of the first two solvation shells. The ratio $g_{\alpha H}(r) / g_{\alpha L}(r)$ reaches values of $g_{\alpha H}(r) / g_{\alpha L}(r) \approx 45$ for distances close to contact. This corresponds to the intuitive picture of preferential solvation that we have given above. The angular contribution of the more polar species, $\left\langle E_{2}^{H} / \mu_{H}\right\rangle(r)$, when defined in this way, actually is smaller than its counterpart corresponding to the less polar species. However, the angular correlation $\left\langle E_{2}^{H}\right\rangle(r)=\left\langle\cos \left(\hat{\boldsymbol{\mu}}^{H} \cdot \hat{\mathbf{r}}\right)\right\rangle$ is larger than $\left\langle E_{2}^{L}\right\rangle(r)$, as expected. The preferential solvation parameter as defined by Marcus ${ }^{21,22}$ is

$$
\delta f_{H}=f_{H}^{\mathrm{loc}}-f_{H},
$$

where $f_{H}^{\text {loc }}$ is the local molar fraction of species $H$ in a sphere of radius $R^{\mathrm{loc}}$ around the ion. Note that this parameter is zero both for the macroscopic (Born) and the MSA description as used in this work. In Fig. 5 we have represented this excess molar fraction for $R^{\text {loc }}=4.0 \AA$, corresponding approximately to the first minimum of $g_{\alpha H}(r)$. The simulation data show a quite large deviation from zero for a wide range of molar fractions. The maximum value of $\delta f_{H} \approx 0.6$ is reached for $f_{H} \approx 0.12$, in agreement with the steep increase of the solvation free energy for small molar fractions, as has been noted above.

The simulations indicate that a better theoretical description of the solvation of hard sphere ions in binary mixtures of hard sphere dipoles must take into account the $r$ dependence of $\hat{h}^{H}\left(r, f_{H}\right) / \hat{h}^{L}\left(r, f_{H}\right)$. Namely, an ion-dipole pair distribu-

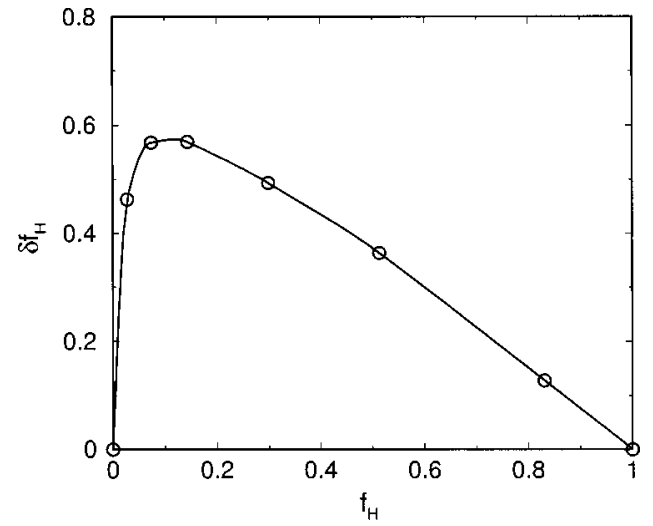

FIG. 5. The preferential solvation parameter as defined in Eq. (63). The local molar fraction has been determined from the radial distribution functions inside a sphere with radius $R^{\mathrm{loc}}=4.0 \AA$ around the solute. The circles represent the simulation results; the solid line is a spline interpolation of these points.

tion function with the form of Eq. (58), which contains separate radial and angular correlation functions for each species, should be used in order to adequately describe the phenomenon of preferential solvation. We will address this question in future work. The approach used here [see Eq. (13)], as well as the theory of Chandra and Bagchi, do not fulfill this requirement. Nevertheless, they reproduce the qualitative behavior of the solvation energy with respect to the molar fraction and represent an improvement over the macroscopic description.

\section{ACKNOWLEDGMENTS}

Support by the Dirección General de Enseñanza Superior of Spain (Project Nos. PB98-0423 and PB98-1120) and the Consejeria de Education y Ciencia of the Junta de Andalucía is gratefully acknowledged.

\section{APPENDIX: MSA RESULTS FOR THE HOMOGENEOUS MIXTURE}

In the paper of Adelman and Deutch, ${ }^{25}$ the direct correlation function for a homogeneous dipole mixture of equally sized molecules of diameter $\sigma$ is given in terms of the MSA solution for the effective fluid

$$
\begin{aligned}
& c_{i j}^{m}(110, k)=\tilde{\mu}^{-2} \mu_{i} \mu_{j} c_{\mathrm{MSA}}^{m}(110, k, \tilde{\rho}, \widetilde{\mu}), \\
& c_{\mathrm{MSA}}^{m}(110, k)=2(4 \pi)^{2} \kappa \int_{0}^{\sigma} d r r^{2} j_{0}(k r) c_{\mathrm{PY}}(r, 2 \kappa \widetilde{\rho}), \\
& c_{\mathrm{PY}}(r)=c_{0}+c_{1}\left(\frac{r}{\sigma}\right)+c_{3}\left(\frac{r}{\sigma}\right)^{3} .
\end{aligned}
$$

Here, $c_{\mathrm{PY}}$ is the Perkus-Yevick direct correlation function at density $2 \kappa \widetilde{\rho}$, and $\kappa$ is obtained from the relation

$$
Q(2 \kappa \widetilde{\eta})-Q(-\kappa \widetilde{\eta})=3 \tilde{y}=3(4 \pi / 9) \beta \widetilde{\rho} \widetilde{\mu}^{2},
$$

with

$$
Q(x)=\frac{(1+2 x)^{2}}{(1-x)^{4}} .
$$


The coefficients $c_{i}$ are

$$
\begin{aligned}
& c_{0}=-Q(\widetilde{\eta}) \equiv-\frac{(1+2 \widetilde{\eta})^{2}}{(1-\tilde{\eta})^{4}}, \\
& c_{1}=6 \widetilde{\eta} \frac{(1+\widetilde{\eta} / 2)^{2}}{1-\tilde{\eta}^{4}}, \\
& c_{3}=\frac{1}{2} \widetilde{\eta} c_{0} .
\end{aligned}
$$

For $k=0$, the correlation function may be expressed in terms of $Q$

$$
c_{\mathrm{MSA}}^{m}(110,0)=\frac{4 \pi}{\tilde{\rho}}(1-Q(2 \kappa \tilde{\eta})) .
$$

The dielectric constant $\epsilon$ of the mixture in the MSA is given by

$$
\epsilon=\frac{Q(2 \kappa \tilde{\eta})}{Q(-\kappa \tilde{\eta})} .
$$

We will define

$$
\widetilde{c}(k)=1-\frac{\tilde{\rho}}{4 \pi} c_{\mathrm{MSA}}^{m}(110, k),
$$

which results as the denominator of the expression for $\gamma_{12}(k)=\gamma_{1}(k)+\gamma_{2}(k)$

$$
X_{11}(k) X_{22}(k)-X_{12}(k) X_{21}(k)=\widetilde{c}(k) .
$$

By noting that $Y_{11}+Y_{22}=\tilde{y}$ and $X_{i i}-X_{i j}=1$, we find that

$\gamma_{12}(k)=\gamma_{1}(k)+\gamma_{2}(k)=\frac{1}{3} \frac{Q(2 \kappa \tilde{\eta})-Q(-\kappa \tilde{\eta})}{\widetilde{c}(k)}$.

In the limiting case $k \rightarrow 0$, we find from Eqs. (A9) and (A10) that

$$
\gamma_{12}(0)=\frac{1}{3}\left(1-\frac{1}{\widetilde{\boldsymbol{\epsilon}}}\right),
$$

for $k \rightarrow \infty, \widetilde{c}(k) \rightarrow 1$ and

$$
\gamma_{12}(k \rightarrow \infty)=\frac{1}{3}(Q(2 \kappa \tilde{\eta})-Q(-\kappa \tilde{\eta})) .
$$

${ }^{1}$ D. Y. C. Chan, D. J. Mitchell, and B. W. Ninham, J. Chem. Phys. 70, 2946 (1979).

${ }^{2}$ A. Chandra and B. Bagchi, J. Chem. Phys. 94, 8367 (1991).

${ }^{3}$ F. Garisto, P. G. Kusalik, and G. N. Patey, J. Chem. Phys. 79, 6294 (1983).

${ }^{4}$ D. V. Matyushov and B. M. Ladanyi, J. Chem. Phys. 110, 994 (1999).

${ }^{5}$ J. K. Hyun and T. Ichiye, J. Chem. Phys. 109, 1074 (1998).

${ }^{6}$ M. Saito and T. Kakitani, Chem. Phys. Lett. 172, 169 (1990).

${ }^{7}$ A. Papazyan and M. Maroncelli, J. Chem. Phys. 95, 9219 (1991).

${ }^{8}$ S. Galera, J. M. Lluch, A. Oliva, and J. Bertran, J. Chem. Soc., Faraday Trans. 88, 3537 (1992).

${ }^{9}$ G. Hummer, L. R. Pratt, and A. E. García, J. Phys. Chem. 100, 1206 (1996).

${ }^{10}$ J.-K. Hyun and T. Ichiye, J. Phys. Chem. B 101, 3596 (1997).

${ }^{11}$ R. M. Lynden-Bell and J. C. Rasaiah, J. Chem. Phys. 107, 1981 (1997).

${ }^{12}$ A. V. Bandura, S. N. Lvov, and D. D. Macdonald, J. Chem. Soc., Faraday Trans. 94, 1063 (1998).

${ }^{13}$ A. Warshel and Z. T. Chu, in Structure and Reactivity in Aqueous Solution. Characterization of Chemical and Biological Systems, ACS Symposium Series, edited by C. J. Cramer and D. G. Truhlar (American Chemical Society, Washington, D. C., 1994), Chap. 6.

${ }^{14}$ A. Chandra, Chem. Phys. Lett. 235, 133 (1995).

${ }^{15}$ R. Olender and A. Nitzan, J. Chem. Phys. 102, 7180 (1995).

${ }^{16}$ T. J. F. Day and G. N. Patey, J. Chem. Phys. 106, 2782 (1997).

${ }^{17}$ F. Cichos, A. Willert, U. Rempel, and C. von Borczyskowski, J. Phys. Chem. 101, 8179 (1997).

${ }^{18}$ A. Yoshimori, T. J. F. Day, and G. N. Patey, J. Chem. Phys. 109, 3222 (1998).

${ }^{19}$ B. Bagchi and R. Biswas, Adv. Chem. Phys. 109, 207 (1999).

${ }^{20}$ C. Denk, M. Morillo, F. Sánchez-Burgos, and A. Sánchez, J. Chem. Phys. 110, 473 (1999).

${ }^{21}$ Y. Marcus, J. Chem. Soc., Faraday Trans. 1 84, 1465 (1988).

${ }^{22}$ Y. Marcus, Pure Appl. Chem. 62, 2069 (1990).

${ }^{23}$ M. S. Wertheim, J. Chem. Phys. 55, 4291 (1971).

${ }^{24}$ L. Blum and W. Fawcett, J. Phys. Chem. 96, 408 (1992).

${ }^{25}$ S. A. Adelman and J. M. Deutch, J. Chem. Phys. 59, 3971 (1973).

${ }^{26}$ C. G. Gray and K. E. Gubbins, Theory of Molecular Liquids (Clarendon, Oxford, 1984).

${ }^{27}$ M. P. Allen and D. J. Tildesley, Computer Simulation of Liquids, 1st ed. (Clarendon, Oxford, 1987).

${ }^{28}$ D. Frenkel and B. Smit, Understanding Molecular Simulation (Academic, San Diego, 1996).

${ }^{29}$ I. G. Tironi, R. Sperb, P. E. Smith, and W. F. van Gunsteren, J. Chem. Phys. 102, 5451 (1995).

${ }^{30}$ G. Hummer, L. R. Pratt, and A. E. Garcia, J. Phys. Chem. 99, 14188 (1995).

${ }^{31}$ G. Hummer and A. Szabo, J. Chem. Phys. 105, 2004 (1996). 